\title{
Avaliação de fatores de risco e antígeno prostático específico no rastreamento de câncer de próstata
}

\author{
Evaluation of risk factors and prostate-specific antigen in prostate cancerscreening
}

\author{
Bárbara Binow Demuner ${ }^{1}$, Linda Christian Carrijo-Carvalho ${ }^{2 *}$ \\ ${ }^{1}$ Acadêmica de Medicina, Centro Universitário do Espírito Santo, UNESC; ${ }^{2}$ Doutora em Ciências, Universidade de são \\ Paulo, USP, Professora do Centro Universitário do Espírito Santo, UNESC
}

\begin{abstract}
Resumo
Introdução: no Brasil, o câncer de maior incidência nos homens é o câncer de próstata (CaP), com 6,9\% de mortalidade. Atualmente, discute-se a aplicabilidade do antígeno prostático específico (PSA) em políticas de rastreamento para CaP e os riscos associados ao sobrediagnóstico. Objetivo: correlacionar a dosagem do PSA com fatores de risco, história clínica e a presença de neoplasia prostática. Metodologia: estudo descritivo transversal que analisou, comparativamente, dados clínico-epidemiológicos e níveis séricos de PSA de 200 pacientes. Valores de PSA foram estratificados em três categorias $(<2,5,2,5-10,0$ e $>10 \mathrm{ng} / \mathrm{ml})$. Resultados: os fatores de risco analisados foram relacionados significativamente com o aumento do PSA e neoplasia prostática. A prevalência de CaP (11\%) e hiperplasia prostática (61\%) foi observada nos pacientes com maior dosagem de PSA, enquanto $1 \%$ dos pacientes apresentou CaP sem alteração do PSA e $4 \%$ tiveram CaP com 2,5-10,0 ng/ml de PSA. Maiores níveis séricos do biomarcador foram relacionados a diabetes (70\%), hipertensão (77\%), uso crônico de medicações (60\%) e ausência de exames periódicos (58\%). O grupo com PSA $>10 \mathrm{ng} / \mathrm{ml}$ teve média de idade maior que o primeiro $(p=0,002)$ e o segundo grupos $(p=0,027)$. Conclusão: a prevalência de hiperplasia prostática benigna associada à alteração do PSA, e o elevado risco de exames falso-positivos evidenciam a preocupação com o sobrediagnóstico. No contexto dos dados clinico-epidemiológicos avaliados, a possibilidade de resultados falso-positivos e falso-negativos associados à dosagem do PSA deve ser considerada, ressaltando a importância de adoção de exames complementares para rastreio do CaP. Palavras-chave: Antígeno Prostático Específico. Biomarcadores. Neoplasia Prostática Intraepitelial. Hiperplasia Prostática. Fatores de Risco.
\end{abstract}

\begin{abstract}
Introduction: in Brazil, the cancer with the highest incidence in men is prostate cancer (PCa), with 6.9\% mortality. Currently, the applicability of prostate specific antigen (PSA) in screening policies for PCa and the risks associated with overdiagnosis are discussed. Objective: to correlate the PSA level with risk factors, clinical history and the presence of prostatic neoplasm. Methods: a cross-sectional descriptive study that analyzed, comparatively, clinical-epidemiological data and serum PSA levels of 200 patients. PSA values were stratified into three categories (<2.5, 2.5-10.0 and $>10 \mathrm{ng} / \mathrm{ml})$. Results: the risk factors analyzed were significantly related to the increase in PSA and prostatic neoplasm. The prevalence of PCa (11\%) and prostatic hyperplasia (61\%) was observed in patients with higher levels of PSA, while $1 \%$ of patients had PCa without PSA changes and $4 \%$ had PCa with $2.5-10.0 \mathrm{ng} / \mathrm{ml}$ PSA. Increased serum levels of the biomarker were related to diabetes (70\%), hypertension (77\%), chronic use of medications (60\%) and periodic exams (58\%). The group with PSA $10 \mathrm{ng} / \mathrm{ml}$ had a mean age greater than the first $(p=0.002)$ and the second group ( $p=0.027)$. Conclusion: the prevalence of benign prostatic hyperplasia associated with PSA change and an increased risk of false-positive tests show a concern with overdiagnosis. In the context of clinical-epidemiological data, the possibility of false-positive and false-negative results associated with the PSA measurement have to be considered, highlighting the importance of complementary tests for PCa screening. Keywords: Prostate-Specific Antigen. Biomarkers. Prostatic Intraepithelial Neoplasia. Prostatic Hyperplasia. Risk Factors.
\end{abstract}

\section{INTRODUÇÃO}

No Brasil, o câncer de próstata (CaP) é o câncer de maior incidência na população masculina e corresponde à segunda causa de morte por câncer nos homens, com taxa de mortalidade de $6,9 \%$ (WHO, 2020). Os fatores de risco identificados para a doença estão relacionados, principalmente à idade, história familiar e etnia. A maioria dos casos diagnosticados ocorre em indivíduos acima de 65 anos (RAWLA, 2019). Considerando-se a idade como prin-

Correspondente/Corresponding: * Linda Christian Carrijo Carvalho Centro Universitário do Espírito Santo (UNESC). - End: Av. Fioravante Rossi, 2930-Martineli. CEP 29703-858. Colatina - ES - Tel.: (11)967075937 -E-mail: Iccarrijo@gmail.com cipal fator de risco para o CaP, o aumento da longevidade na população e a inversão da pirâmide etária são fatores preocupantes que devem ser considerados nas políticas públicas e serviços de saúde, para promoção de cuidados na prevenção e no diagnóstico precoce do câncer.

O tumor in situ, geralmente, não é acompanhado de sintomas ou manifestações clínicas, e pode progredir lentamente ao longo da vida do indivíduo, sem causar morbidade. Ao contrário do câncer localizado, processos tumorais invasivos com metástase óssea, por exemplo, tem pior prognóstico e não são facilmente tratáveis. 0 diagnóstico é baseado principalmente no exame digital retal, dosagem do antígeno prostático específico (PSA) e 
biópsia guiada por ultrassonografia transretal, embora o rastreamento pelo toque retal e PSA seja controverso (GROSSMAN et al., 2018; LESLIE et al., 2021). Não há um consenso sobre o limiar de PSA para indicar a biópsia e direcionar o diagnóstico de CaP. Comumente, utiliza-se valores acima de $4 \mathrm{ng} / \mathrm{ml}$, porém estudos têm demonstrado maior sensibilidade na detecção de CaP considerando valores superiores a $2,5 \mathrm{ng} / \mathrm{ml}$ (GILBERT et al., 2005; SAITO, 2007).

Atualmente, recomenda-se que a triagem periódica seja optativa em homens de 55 a 69 anos, com base na decisão médica e do paciente, considerando a história familiar, fatores de risco individuais, bem como potenciais riscos e benefícios associados ao diagnóstico (SBU, 2018). Argumentos a favor da adoção de programas de rastreamento visam diminuir a mortalidade e morbidade associada aos casos com pior evolução clínica. Estima-se que, a partir da dosagem do PSA, é possível evitar duas mortes para cada 1000 homens submetidos ao rastreamento (GROSSMAN et al., 2018).

Este trabalho teve como objetivo analisar a variabilidade dos valores de PSA e correlaciona-los com potenciais fatores de risco como idade, presença de comorbidades, hiperplasia prostática e CaP. Dessa forma, são apresentadas características clínico-epidemiológicas associadas à alteração dos níveis séricos de PSA a fim de avaliar a aplicabilidade deste biomarcador.

\section{METODOLOGIA}

Trata-se de um estudo descritivo transversal, que foi realizado a partir dos dados obtidos de 200 pacientes do sexo masculino em um Hospital Estadual do Município de Colatina - ES. O estudo foi aprovado pelo Comitê de Ética em Pesquisa Institucional sob o número do parecer 3.046.977, conforme resolução de $n^{\circ} 466$, de 12 de dezembro de 2012 do Conselho Nacional de Saúde.

Níveis séricos de PSA, quantificados a partir de amostras de sangue de forma automatizada pelo método de quimiluminescência, foram correlacionados com dados clínico-epidemiológicos dos pacientes. Foram analisados dados sobre idade, etnia, estado civil, realização de exames periódicos, uso de medicação, história familiar, história clínica, estado geral de saúde (higidez), hiperplasia prostática, $\mathrm{CaP}$, bem como a presença de comorbidades como hipertensão arterial sistêmica (HAS), diabetes mellitus e outras doenças.

As análises comparativas foram realizadas considerando-se três grupos estratificados a partir da dosagem de PSA, conforme Gilbert et al. (2005), nos grupos I (<2,5 ng/ $\mathrm{ml})$, II (2,5 a 10,0 ng/ml), e III (> $10 \mathrm{ng} / \mathrm{ml})$. Os resultados foram expressos em valores percentuais associados às variáveis categóricas e numéricas. Para análises estatísticas, utilizou-se análise da variância (ANOVA) seguida pelo teste de Tukey para variáveis numéricas e o teste de Fisher para variáveis categóricas, considerando-se significantes valores de $p<0,05$.

\section{RESULTADOS}

Um total de 200 pacientes foram estratificados, conforme a quantificação do PSA, nos grupos G-I $(<2,5 \mathrm{ng} /$ $\mathrm{ml}), \mathrm{G}-\mathrm{II}(2,5$ a $10,0 \mathrm{ng} / \mathrm{ml}$ ) e G-III (> $10 \mathrm{ng} / \mathrm{ml})$, nos quais foram observados um total de 88,69 e 43 pacientes, respectivamente. A dosagem de PSA mensurada nesses grupos correspondeu a $1,9 \pm 0,4 \mathrm{ng} / \mathrm{ml} \mathrm{em} \mathrm{G-I} \mathrm{(} 44 \%$ do total de pacientes analisados), $5,2 \pm 1,4 \mathrm{ng} / \mathrm{ml} \mathrm{em} \mathrm{G-II}$ (34,5\% dos pacientes) e $11,2 \pm 1,9 \mathrm{ng} / \mathrm{ml}$ em G-III (21,5\% dos pacientes), conforme demonstrado na Figura 1.

A Figura 2 mostra a distribuição da idade nos diferentes grupos conforme a dosagem de PSA. A idade média correspondeu a $61 \pm 4,6$ anos no grupo I, $62 \pm 5,5$ no grupo II e $64 \pm 4,6$ no grupo III. O grupo com PSA $>10 \mathrm{ng} / \mathrm{ml}$ apresentou idade significantemente maior que o primeiro $(p=0,002)$ e o segundo grupo $(p=0,027)$.

Figura 1 - Níveis séricos de PSA em 200 pacientes do sexo masculino estratificados em três grupos, de acordo com a dosagem de PSA. G-I: <2,5 ng/ml, G-II: 2,5 a 10,0 ng/ml, e G-III: $>10 \mathrm{ng} / \mathrm{ml}$.

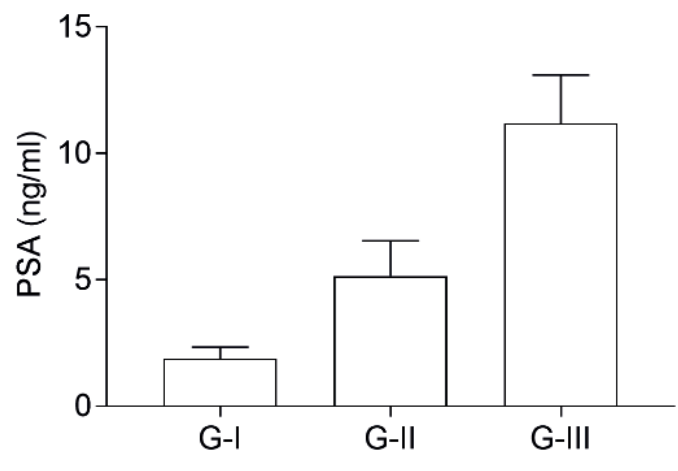

Fonte: Autoria própria

Figura 2 - Distribuição da idade em 200 pacientes do sexo masculino com diferentes níveis séricos de PSA.

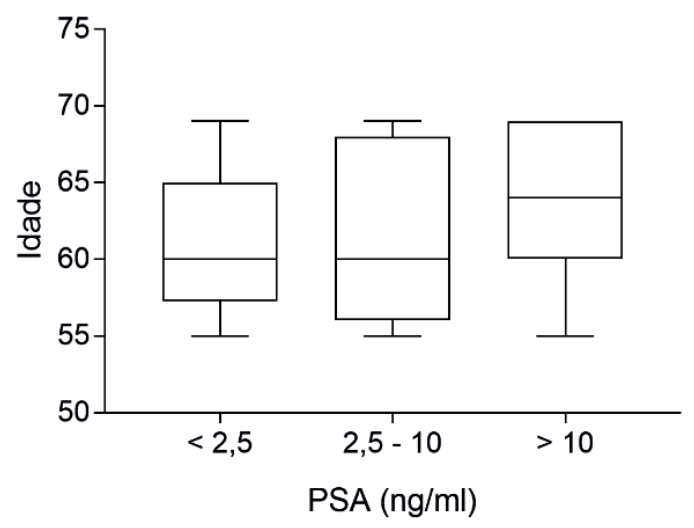

Fonte: Autoria própria 
A prevalência dos fatores de risco nos pacientes estratificados nos três grupos conforme os níveis séricos de PSA está demonstrada na Tabela 1. Maiores medidas de PSA (>10 ng/ml) foram correlacionadas, de forma significante, com história familiar positiva de CaP (88\%), afrodescendência (42\%), ausência de exames periódicos, hipertensão arterial sistêmica (HAS, 77\%) e diabetes (70\%). Também houve prevalência estatisticamente significante desses fatores de risco no G-II, associada a níveis de PSA moderadamente elevados, excetuando-se afrodescendência e HAS. No G-II, o uso crônico de medicações e o estado civil solteiro ou divorciado também se mostraram como fatores de risco com prevalência significantemente maior.

A Tabela 2 apresenta a correlação dos níveis séricos de PSA com diagnósticos clínicos relacionados a patologia prostática e estado de saúde dos pacientes. Apenas um paciente com CaP foi observado no G-I, com $2,4 \mathrm{ng} / \mathrm{ml}$ de PSA, 69 anos, afrodescendente, portador de diabetes, HAS e em uso crônico de medicações. No G-II, os três pacientes com CaP apresentaram PSA de 7,0 a 8,5 ng/ml, idade entre 68 e 69 anos, história familiar positiva, HAS e não eram casados. No G-III, observou-se medida do PSA entre 10,0 e $13,0 \mathrm{ng} / \mathrm{ml}, 66$ a 69 anos de idade e HAS em todos os pacientes. A prevalência de $\mathrm{CaP}$ foi cerca de 3 vezes maior no G-III em relação ao G-II e 10 vezes maior em relação ao G-I. No G-III, $12 \%$ dos pacientes apresentaram CaP, cuja diferença foi estatisticamente significante em relação ao G-1 com níveis séricos normais de PSA. O G-III também apresentou a maior proporção dos pacientes com hiperplasia prostática benigna (60\%) em comparação a G-I e G-II, com prevalência quase duas vezes maior. Os grupos G-I e G-II, com nível baixo e moderadamente elevado de PSA, respectivamente, apresentaram $65 \%$ dos pacientes hígidos, em contrapartida ao grupo com PSA $>10 \mathrm{ng} / \mathrm{ml}$, que apresentou decréscimo significante de pacientes hígidos. Apenas um paciente (1\%) apresentou CaP sem alteração do PSA e três pacientes (4\%) tiveram CaP com $2,5-10,0 \mathrm{ng} / \mathrm{ml}$ de PSA.

Tabela 1 - Prevalência de fatores de risco associados a diferentes níveis séricos de PSA em 200 pacientes do sexo masculino.

\begin{tabular}{|c|c|c|c|c|c|c|}
\hline Níveis de PSA & $\begin{array}{c}\text { G-I1 } \\
\text { n (\%) }\end{array}$ & $\begin{array}{c}\mathbf{P}^{*} \\
\text { | e II }\end{array}$ & $\begin{array}{c}\text { G-II2 } \\
\text { n (\%) }\end{array}$ & $\begin{array}{c}P \\
\text { II e III }\end{array}$ & $\begin{array}{l}\text { G-III3 } \\
\text { n (\%) }\end{array}$ & $\begin{array}{c}P \\
\text { I e III }\end{array}$ \\
\hline História familiar positiva & $55(63,6)$ & 0,0206 & $56(81,1)$ & 0,4295 & $38(88,4)$ & 0,0035 \\
\hline Afrodescendente & $14(15,9)$ & 0,1618 & $18(26,1)$ & 0,0982 & $18(41,8)$ & 0,0021 \\
\hline Casado & $62(70,5)$ & 0,0348 & $59(85,5)$ & 0,0560 & $30(69,8)$ & $>0,9999$ \\
\hline Realiza exames periódicos & $61(69,3)$ & 0,0019 & $30(43,5)$ & 0,9999 & $18(41,9)$ & 0,0041 \\
\hline Uso crônico medicações & $37(42,1)$ & 0,0098 & $44(63,7)$ & 0,8413 & $26(60,5)$ & 0,0626 \\
\hline HAS & $49(55,7)$ & 0,0979 & $48(69,6)$ & 0,5160 & $33(76,7)$ & 0,0218 \\
\hline Diabetes & $41(46,6)$ & 0,0240 & $45(65,2)$ & 0,6827 & $30(69,7)$ & 0,0153 \\
\hline
\end{tabular}

${ }^{1}$ Grupo com PSA $<2,5 ;{ }^{2}$ Grupo com PSA 2,5-10,0; ${ }^{3}$ Grupo com PSA $>10,0$.

*Comparação entre os grupos, valores de P obtidos pelo teste de Fisher.

Fonte: Dados da pesquisa

Tabela 2 - História clínica associada a diferentes níveis séricos de PSA em 200 pacientes do sexo masculino.

\begin{tabular}{l|c|c|c|c|c|c}
\hline \multicolumn{1}{c|}{ Níveis de PSA } & $\begin{array}{c}\text { G-I1 } \\
\mathbf{n}(\%)\end{array}$ & $\begin{array}{c}\text { P* } \\
\text { I e II }\end{array}$ & $\begin{array}{c}\text { G-II2 } \\
\text { n (\%) }\end{array}$ & $\begin{array}{c}\text { P } \\
\text { II e III }\end{array}$ & $\begin{array}{c}\text { G-III3 } \\
\text { n (\%) }\end{array}$ & $\begin{array}{c}\text { P } \\
\text { I e III }\end{array}$ \\
\hline CaP & $1(1,1)$ & 0,3205 & $3(4,3)$ & 0,2563 & $5(11,6)$ & 0,0145 \\
\hline Hiperplasia prostática & $30(34,1)$ & 0,7317 & $21(30,4)$ & 0,0029 & $26(60,4)$ & 0,0050 \\
\hline Outras doenças & $30(34,1)$ & 0,8647 & $22(31,8)$ & 0,0101 & $25(58,1)$ & 0,0138 \\
\hline Paciente hígido & $57(64,8)$ & 0,9999 & $45(65,2)$ & 0,0002 & $12(27,9)$ & 0,0001 \\
\hline
\end{tabular}

${ }^{1}$ Grupo com PSA $<2,5 ;{ }^{2}$ Grupo com PSA 2,5 - 10,0; ${ }^{3}$ Grupo com PSA $>10,0$.

*Comparação entre os grupos, valores de $P$ obtidos pelo teste de Fisher.

Fonte: Dados da pesquisa

\section{DISCUSSÃO}

O CaP apresenta ampla variabilidade clínica e se manifesta, frequentemente, como uma doença assintomática nos estágios iniciais (LESLIE et al., 2021; LITWIN; TAN, 2017). A importância do diagnóstico precoce e a dificuldade de adesão de muitos homens em realizar exames por via retal (COSTA et al., 2020), tornam a utilização de biomarcadores desejável na prática clínica. O PSA é o marcador sérico mais utilizado atualmente para rastreamento do CaP, e sua aplicabilidade tem sido amplamente discutida na literatura (KENSLER et al., 2021; MODESTO et al., 2018; TABAYOYONG; ABOUASSALY, 2015).

Diferentes autores demonstraram maior sensibilidade na utilização de um valor limiar de PSA até $2,5 \mathrm{ng} / \mathrm{ml}$ do que o valor de referência comumente utilizado, abaixo de 4 ng/ml (GILBERT et al., 2005; SAITO, 2007). Quando 
há o acompanhamento com realização de exames periódicos, é possível mensurar a velocidade do PSA, onde se observa o aumento da concentração do biomarcador superior a $0,35 \mathrm{ng} / \mathrm{ml}$ ao ano associado ao CaP (CARTER et al., 2006). A elevação do PSA também pode estar associada ao aumento do volume prostático em condições de hiperplasia prostática benigna, onde há uma relação do volume prostático com o aumento da medida do PSA (CATALONA et al., 1998).

Neste trabalho, um total de 200 pacientes foram avaliados, associando-se a medida do PSA com patologia prostática benigna ou maligna e potenciais fatores de risco. Trata-se de um estudo pioneiro de abordagem clínico-epidemiológica de neoplasias prostáticas e fatores de risco associados ao aumento do PSA na população da região noroeste do Espírito Santo. A maioria dos pacientes foi estratificada no G-I, com PSA $<2,5 \mathrm{ng} / \mathrm{ml}$, para o qual foi observado apenas um resultado falso-negativo no diagnóstico de $\mathrm{CaP}$ dependente da medida do PSA. Por outro lado, os resultados mostraram muitos resultados falso-positivos de CaP nos demais grupos, com elevação do PSA não associada a malignidade. Nesses grupos houve alta prevalência de hiperplasia prostática benigna. Com exceção do paciente com CaP observado no G-I, os casos positivos de $\mathrm{CaP}$ observados neste trabalho se enquadraram no limiar de referência comumente utilizado, acima de $4 \mathrm{ng} /$ $\mathrm{ml}$, apresentando valores maiores que $7 \mathrm{ng} / \mathrm{ml}$ de PSA.

Os principais fatores de risco reconhecidos para o CaP pela American Cancer Society (2020) e reportados na literatura incluem idade maior que 40 anos; etnia afro-americana e afro-caribenha; localização geográfica na América do Norte, noroeste da Europa, Austrália e ilhas do Caribe; história familiar; mutações (ex. gene BRCA2) e polimorfismos genéticos (GROZESCU; POPA, 2017; PERNAR et al., 2018; REBBECK, 2017). A redução da ingesta de gordura e aumento do consumo de proteína de soja, licopeno, vitamina $D$, vitamina $E$ e suplementação com selênio são considerados fatores protetores (MASKO; ALLOTT; FREEDLAND, 2013; RAWLA, 2019; SANTILLO; LOWE, 2006).

Os dados obtidos no presente estudo corroboram com dados previamente publicados, demonstrando a relevância dos fatores de risco conhecidos para o $\mathrm{CaP}$, e sugerem outros fatores que podem ser considerados em associação ao aumento dos níveis de PSA. Possivelmente, tais fatores em conjunto contribuem para o desenvolvimento de neoplasias prostáticas benignas e malignas. Os resultados sugerem que tanto o $\mathrm{CaP}$, como a hiperplasia prostática benigna estão associados ao grupo de pacientes com maior prevalência de comorbidades. A menor realização de exames periódicos e o uso crônico de medicações também foram apontados como possíveis fatores associados à elevação do PSA, os quais poderiam ser fatores de risco para o CaP. A HAS também foi observada como um importante fator de risco neste estudo, presente em todos os pacientes que apresentaram $\mathrm{CaP}$, independente da dosagem do PSA.
O método padrão ouro para rastreio consiste no toque retal. A dosagem de PSA é adotada como exame laboratorial complementar de rastreio, embora, em muitos casos seja o método de avaliação principal em pacientes que não apresentam adesão ao toque retal por motivos psicossociais. De acordo com a Sociedade Brasileira de Urologia (2018), a aplicabilidade clínica do PSA é controversa devido à baixa especificidade deste biomarcador, principalmente nas concentrações entre 2 e $10 \mathrm{ng} / \mathrm{ml}$.

Neste estudo, observou-se que, de fato o PSA apresenta um baixo valor diagnóstico para o CaP, podendo estar elevado em outras condições, como a hiperplasia prostática benigna. Porém, o uso deste biomarcador associado ao exame de toque retal pode ser uma importante ferramenta na identificação precoce do CaP, e na identificação dos pacientes que necessitam de acompanhamento clínico mais frequente e do monitoramento de neoplasias prostáticas através de exames periódicos.

\section{CONCLUSÃO}

Os resultados obtidos podem contribuir para o avanço da medicina personalizada, na consideração dos fatores de risco e diagnóstico precoce de neoplasias de próstata. O PSA mostrou-se um marcador pouco específico para o rastreamento do CaP se considerado isoladamente, e pode contribuir a fim de se evitar problemas de sobrediagnóstico associado a biópsia.

\section{AGRADECIMENTOS}

Os autores agradecem ao Programa Institucional de Bolsas de Iniciação Científica e Tecnológica do Centro Universitário do Espírito Santo (PIBICT-UNESC) pela concessão de bolsa de Iniciação Científica a um dos autores (B.B.D.).

\section{REFERÊNCIAS}

AMERICAN CANCER SOCIETY. Prostate Cancer Risk Factors. 2020. Disponível em: https://www.cancer.org/cancer/prostate-cancer/causesrisks-prevention/risk-factors.html. Acesso em: 26 Oct. 2020.

CARTER, H. B. et al. Detection of life-threatening prostate cancer with prostate-specific antigen velocity during a window of curability. J. Natl. Cancer Inst., Cary, v. 98, n. 21, p. 1521-1527, 2006.

CATALONA, W. J. et al. Use of the percentage of free prostate-specific antigen to enhance differentiation of prostate cancer from benign prostatic disease: a prospective multicenter clinical trial. Jama, Rio de Janeiro, v. 279, n. 19, p. 1542, 1998.

COSTA, N. M. P. C. et al. O exame de toque retal e o olhar masculino. Rev. Bra. Edu. Saúde, [s.I], v. 10, n.1, p. 143-149, 2020.

GILBERT, S. M. et al. Evidence suggesting PSA cutpoint of $2.5 \mathrm{ng} / \mathrm{mL}$ for prompting prostate biopsy: review of 36,316 biopsies. Urology, Ridgewood, v. 65, n. 3, p. 549-553, 2005.

GROSSMAN, D. C. et al. Screening for Prostate Cancer: US Preventive Services Task Force Recommendation Statement. Jama, Rio de Janeiro, v. 319, n.18, p. 1901-1913, 2018.

GROZESCU, T.; POPA, F. Prostate cancer between prognosis and adequate/proper therapy. J. Med. Life, [s.I], v. 10, n. 1, p. 5-12, 2017. 
KENSLER, K. H. et al. Racial and ethnic variation in PSA testing and prostate cancer incidence following the 2012 U.S.P.S.T.F. recommendation. J. Natl. Cancer Inst., Caryv, v.113, n. 6, 2021.

LESLIE, S. W. et al. Prostate cancer. In: STATPEARLS. Treasure Island (FL): StatPearls Publishing, 2021. Disponível em: https://pubmed.ncbi.nlm. nih.gov/29261872/. Acesso em: 20 Mar. 2021.

LITWIN, M. S.; TAN, H. J. The Diagnosis and treatment of prostate cancer: a review. Jama, Chicago, v. 317, n. 24, p. 2532-2542, 2017.

MASKO, E. M.; ALLOTT, E. H.; FREEDLAND, S. J. The relationship between nutrition and prostate cancer: is more always better? Eur. Urol., Basel, v. 63, n. 5, p.810-820, 2013.

MODESTO, A. A. D. A. et al. Um novembro não tão azul: debatendo rastreamento de câncer de próstata e saúde do homem. Interface (Botucatu), Botucatu, v. 22, n. 64, p. 251-262, 2018.

PERNAR, C. H. et al. The Epidemiology of Prostate Cancer. Cold Spring Harb Perspect. Med., [s.l], v. 8, n. 12, p. a030361, 2018.

RAWLA, P. Epidemiology of Prostate Cancer. World J. Oncol., London, v. 10, n. 2, p. 63-89, 2019.

REBBECK, T. R. Prostate Cancer Genetics: Variation by Race, Ethnicity, and
Geography. Semin. Radiat. Oncol., Philadelphia, v. 27, n. 1, p. 3-10, 2017.

SAITO, S. Prostate-specific antigen cut-off point of $2.5 \mathrm{ng} / \mathrm{mL}$ and increasing the number of prostate biopsies results in the detection of curable prostate cancer even in Japanese population. Int. J. Urol., Tokyo, v. 14, n. 8, p. 709-712, 2007.

SANTILLO, V. M; LOWE, F. C. Role of vitamins, minerals and supplements in the prevention and management of prostate cancer. Int. Braz. J. Urol., Rio de Janeiro, v. 32, n. 1, p. 3-14, 2006.

SOCIEDADE BRASILEIRA DE UROLOGIA (SBU). Posicionamento Oficial da Sociedade Brasileira de Patologia Clínica/Medicina Laboratorial (SBPC/ML) e da Sociedade Brasileira de Urologia (SBU) - Rastreio de Câncer de Próstata. 2018. Disponível em: http://www.sbpc.org.br/ wpcontent/uploads/2018/11/SBPC_PSA_posicionamento_final-1.pdf. Acesso em: 04 dez. 2020.

TABAYOYONG, W; ABOUASSALY, R. Prostate Cancer Screening and the Associated Controversy. Surg. Clin. North Am., Philadelphia, v. 95, n. 5, p. 1023-1039, 2015.

WORLD HEALTH ORGANIZATION (WHO). Cancer country profiles 2020. Disponível em: https://www.who.int/cancer/country-profiles/Global_ Cancer_Profile_2020.pdf. 2020. Acesso em: 25 Nov. 2020.

Submetido em: $28 / 03 / 2021$

Aceito em: 08/06/2021 